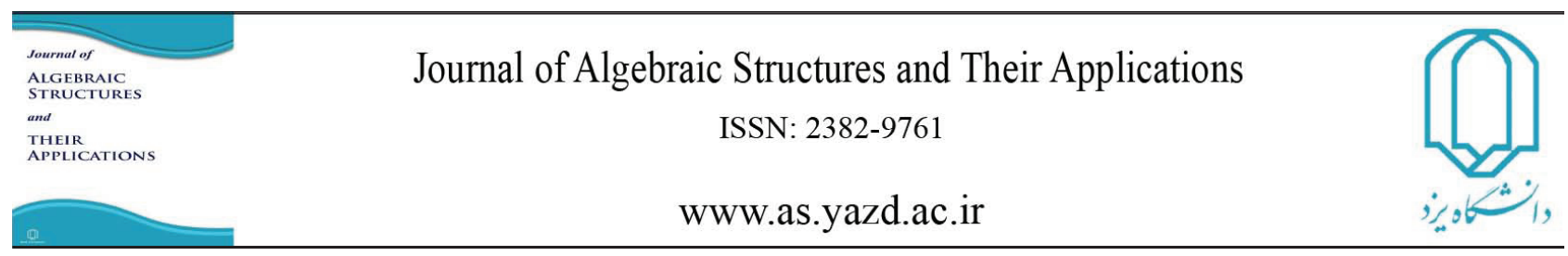

Algebraic Structures and Their Applications Vol. 5 No. 2 ( 2018 ) pp 35-43.

\title{
FINITE GROUPS ADMITTING A CONNECTED CUBIC INTEGRAL BI-CAYLEY GRAPH
}

\author{
MAJID AREZOOMAND* AND BIJAN TAERI \\ Communicated by M.A. Iranmanesh
}

\begin{abstract}
A graph is called integral if all eigenvalues of its adjacency matrix are integers. Given a subset $S$ of a finite group $G$, the bi-Cayley graph $\operatorname{BCay}(G, S)$ is a graph with vertex set $G \times\{1,2\}$ and edge set $\{\{(x, 1),(s x, 2)\} \mid s \in S, x \in G\}$. In this paper, we classify all finite groups admitting a connected cubic integral bi-Cayley graph.
\end{abstract}

\section{INTRODUCTION AND RESULTS}

A graph is called integral if its adjacency matrix has integer eigenvalues. Harary and Schwenk [9] suggested the problem of determining which graphs are integral. This problem has been attacked by many algebraic graph theorists during the last forty years and it is still open. In general the problem seems too difficult. So some people investigated special classes of graphs, including graphs of bounded valency and Cayley graphs. Schwenk [1:3] proved that there are exactly thirteen connected cubic integral graphs. At the same time, independently, the similar result was reported by Bussemaker and Cvetković [7]. The problem of classifying

DOI :http://dx.doi.org/10.29252/asta.5.2.35

MSC(2010): 05C50, 20C10 Primary:05C25, 05E10

Keywords: Bi-Cayley graph, Integer eigenvalues, Irreducible representation.

Received: 22 March 2018, Accepted: 16 November 2018.

*Corresponding author

(C) 2018 Yazd University. 
and constructing integral Cayley graphs was started by Abdollahi and Vatandoost [ד]. They classified all finite groups admitting a connected cubic integral Cayley graph. These are finite groups $G$ with the property that whenever $S=S^{-1} \subseteq G \backslash\{1\}$ is a 3-element generating set for $G$, the Cayley graph of $G$ with respect to $S$, denoted by Cay $(G, S)$, is an integral graph.

The bi-Cayley graph of a group $G$ with respect to a subset $S \subseteq G$, denoted by $\operatorname{BCay}(G, S)$, is a graph with vertex set $G \times\{1,2\}$ and edge set $\{\{(x, 1),(s x, 2)\} \mid x \in G, s \in S\}$. The bi-Cayley graph $\operatorname{BCay}(G, S)$ is a bipartite $|S|$-regular graph. Also, by [П1, p. 1259], it is connected if and only if $G=\left\langle S S^{-1}\right\rangle$. Furthermore, if $1 \in S$ then $\operatorname{BCay}(G, S)$ is connected if and only if $G=\langle S\rangle$. Also if $S=S^{-1}$ then $\operatorname{BCay}(G, S)$ is isomorphic to the tensor product Cay $(G, S) \otimes K_{2}$ [4, Lemma 3.2]. Therefore if $S=S^{-1}$, then BCay $(G, S)$ is integral if and only if Cay $(G, S)$ is integral. But in general, the connectivity of $\operatorname{BCay}(G, S)$ is not equivalent to the connectivity of $\operatorname{Cay}(G, S)$.

The Cayley graphs and bi-Cayley graphs are special classes of $n$-Cayley graphs. For a positive integer $n$, a graph $\Gamma$ is called $n$-Cayley graph over a group $G$ if the full automorphism group of $\Gamma$ has a semiregular subgroup isomorphic to $G$ with $n$ orbits. Recently, the present authors determined the eigenvalues of $n$-Cayley graphs in [ [2]. By [2, Lemma 2], an $n$-Cayley graph is characterized by $n^{2}$ subsets $T_{i, j}, 1 \leq i, j \leq n$, of $G$ (some subsets may be empty). An $n$-Cayley graph over a group $G$ can be identified by a graph $\Gamma=\operatorname{Cay}\left(G, T_{i, j} \mid 1 \leq i, j \leq n\right)$, where $T_{i, j}$ 's are subsets of $G, V(\Gamma)=G \times\{1, \ldots, n\}$ and $(x, i)$ is adjacent to $(y, j)$ if and only if $y x^{-1} \in T_{i, j}$. It follows that every bi-Cayley graph is a 2-Cayley graph and $\operatorname{BCay}(G, S) \cong$ $\operatorname{Cay}\left(G, T_{1,1}, T_{2,2}, T_{1,2}, T_{2,1}\right)$, where $T_{1,1}=T_{2,2}=\emptyset, T_{1,2}=S$ and $T_{2,1}=S^{-1}$. Also by the definition of Cayley graphs, every Cayley graph is a 1-Cayley graph.

Let $G$ be a finite group, $\operatorname{irr}(G)=\left\{\rho_{1}, \ldots, \rho_{m}\right\}$ and $\operatorname{Irr}(G)=\left\{\chi_{1}, \ldots, \chi_{m}\right\}$ be the set of all irreducible inequivalent $\mathbb{C}$-representations and $\mathbb{C}$-characters of $G$, respectively. If $G$ is abelian, then $\operatorname{Irr}(G)=\operatorname{irr}(G)$. For a subset $X$ of $G$ and $l=1, \ldots, m$, we set $\rho_{l}(X):=\sum_{x \in X} \rho_{l}(x)$. We use these notation throughout the paper. Note that for each irreducible character $\chi$ of $G$ and each $g \in G, \chi\left(g^{-1}\right)$ is the complex conjugate of $\chi(g)$.

A result of Diaconis and Shahshahani [ 8$]$ shows that there is a link between the representation theory and spectral theory of Cayley graphs. From $[8]$ one can deduce that $\operatorname{Cay}(G, S)$ is integral if and only if $\rho(S)$ has integer eigenvalues, for all $\rho \in \operatorname{irr}(G)$, see also [2, Corollary 7]. In particular, if $G$ is abelian then $\operatorname{Cay}(G, S)$ is integral if and only if $\chi(S)$ is an integer, for all $\chi \in \operatorname{Irr}(G)$. The present author and Taeri represented the adjacency matrix of an $n$-Cayley graph over $G$ as a diagonal block matrix in terms of irreducible representations of $G$ and determined its characteristic polynomial in [2]. Using of [Z, Theorem 6], we correlate the integrality of bi-Cayley graph to the irreducible representations of $G$ in the following theorem: 
Theorem 1.1. Let $A$ be the adjacency matrix of $\Gamma=\operatorname{BCay}(G, S), \operatorname{irr}(G)=\left\{\rho_{1}, \ldots, \rho_{m}\right\}$ and

$$
A_{l}=\left[\begin{array}{cc}
0_{d_{l}} & \rho_{l}\left(S^{-1}\right) \\
\rho_{l}(S) & 0_{d_{l}}
\end{array}\right],
$$

where $d_{l}$ is the degree of $\rho_{l}$ and $0_{d_{l}}$ is the $d_{l} \times d_{l}$ matrix with all entries 0 . Then

(1) there exists a basis $B$ such that $[A]_{B}=\operatorname{Diag}\left(I_{d_{1}} \otimes A_{1}, \ldots, I_{d_{m}} \otimes A_{m}\right)$.

(2) $p_{A}(\lambda)=\prod_{l=1}^{m} p_{A_{l}}(\lambda)^{d_{l}}$, where $p_{X}(\lambda)$ is the characteristic polynomial of matrix $X$.

(3) $\Gamma$ is integral if and only if for every $l=1, \ldots, m, A_{l}$ has integer eigenvalues.

In particular, if $G$ is an abelian group then eigenvalues of $\Gamma=\operatorname{BCay}(G, S)$ are $\pm|\chi(S)|$, where $\chi \in \operatorname{Irr}(G)$. Also $\Gamma$ is integral if and only if $|\chi(S)| \in \mathbb{Z}$, for all $\chi \in \operatorname{Irr}(G)$.

The aim of this paper is to classify finite groups admitting a connected cubic integral biCayley graph. For the group-theoretic and graph-theoretic terminology not defined here we refer the reader to [10], [5], respectively. We use the symbols $\mathbb{Z}_{n}, D_{2 n}, S_{n}, A_{n}$, and Dic 12 for the cyclic group of order $n$, the dihedral group of order $2 n$, the symmetric group on $n$ letters, the alternating group on $n$ letters, and the dicyclic group of order 12 , respectively. Also $\lambda^{[k]}$ denotes that $\lambda$ is an eigenvalue of multiplicity $k$.

We use the following theorem frequently, which is well-known in the representation theory of finite groups.

Theorem 1.2. (1) Let $G=\langle a\rangle \cong \mathbb{Z}_{n}$ and $\omega=\exp (2 \pi i / n)$. Then $\operatorname{Irr}(G)=\left\{\chi_{r} \mid 0 \leq r \leq\right.$ $n-1\}$, where $\chi_{r}\left(a^{s}\right)=\omega^{r s}, 0 \leq r, s \leq n-1$.

(2) Let $G=\left\langle r, s \mid r^{n}=s^{2}=(r s)^{2}=1\right\rangle \cong D_{2 n}$. Then for odd $n$ the irreducible representations of $G$ are

$$
\begin{aligned}
& \rho_{1}: s^{v} r^{u} \mapsto 1, \\
& \rho_{2}: s^{v} r^{u} \mapsto(-1)^{v}, \\
& \psi_{k}: r^{u} \mapsto\left[\begin{array}{cc}
\omega^{k u} & 0 \\
0 & \omega^{-k u}
\end{array}\right], s r^{u} \mapsto\left[\begin{array}{cc}
0 & \omega^{-k u} \\
\omega^{k u} & 0
\end{array}\right], \quad 1 \leq k \leq(n-1) / 2,
\end{aligned}
$$

and for even $n$ the irreducible representations of $G$ are

$$
\begin{aligned}
& \rho_{1}: s^{v} r^{u} \mapsto 1, \\
& \rho_{2}: s^{v} r^{u} \mapsto(-1)^{v}, \\
& \rho_{3}: s^{v} r^{u} \mapsto(-1)^{u}, \\
& \rho_{4}: s^{v} r^{u} \mapsto(-1)^{u+v}, \\
& \psi_{k}: r^{u} \mapsto\left[\begin{array}{cc}
\omega^{k u} & 0 \\
0 & \omega^{-k u}
\end{array}\right], s r^{u} \mapsto\left[\begin{array}{cc}
0 & \omega^{-k u} \\
\omega^{k u} & 0
\end{array}\right], \quad 1 \leq k \leq n / 2-1,
\end{aligned}
$$


where $\omega=\exp (2 \pi i / n)$.

(3) Every irreducible character of $G \times H$ is of the form $\chi \times \mu$, where $\chi \in \operatorname{Irr}(G), \mu \in \operatorname{Irr}(H)$ and $(\chi \times \mu)(g, h)=\chi(g) \mu(h)$.

Let $C_{n}$ be a cycle with $n$ vertices. It is well-known that the eigenvalues of $C_{n}$ are $2 \cos (2 \pi j / n), j=0,1, \ldots, n-1$. Hence, one can easily see that the only integral cycles are ones with 3,4 or 6 vertices. In the following proposition, we find all groups admitting a connected 2-regular integral bi-Cayley graph.

Proposition 1.3. Let $G$ be a finite group. Then $G$ admits a connected integral bi-Cayley graph of valency 2 if and only if $G \cong \mathbb{Z}_{2}$ or $\mathbb{Z}_{3}$.

Proof. Let $S$ be a subset of $G,|S|=2$ and $\operatorname{BCay}(G, S)$ be connected and integral. So $\operatorname{BCay}(G, S) \cong C_{2|G|}$ is integral and therefore $|G|=2$ or 3 . Conversely, let $G_{1}=\langle a\rangle \cong \mathbb{Z}_{2}$, $G_{2}=\langle b\rangle \cong \mathbb{Z}_{3}, S_{1}=G_{1}$ and $S_{2}=\{1, b\}$. Then it is easy to see that BCay $\left(G_{1}, S_{1}\right) \cong C_{4}$ and $\operatorname{BCay}\left(G_{2}, S_{2}\right) \cong C_{6}$, which both are connected and integral.

In the following theorem, we classify all finite groups admitting a connected cubic integral bi-Cayley graph.

Theorem A. A finite group $G$ admits a connected cubic integral bi-Cayley graph if and only if $G$ is isomorphic to one of the groups

$$
\mathbb{Z}_{2}^{2}, \mathbb{Z}_{3}, \mathbb{Z}_{4}, \mathbb{Z}_{6}, \mathbb{Z}_{2} \times \mathbb{Z}_{6}, S_{3}, A_{4}, \text { Dic }_{12}
$$

We need the following lemma which yields that if $G$ admits a connected integral bi-Cayley graph of valency $k$, then $\mathbb{Z}_{2} \ltimes G$ admits a connected integral Cayley graph of valency $k$.

Lemma 1.4. (see [3, Lemma 4.8]) Let $G$ be a finite abelian group and $\Gamma=\operatorname{BCay}(G, S)$ be a bi-Cayley graph of $G$. Then $\Gamma \cong \operatorname{Cay}\left(\langle\psi\rangle R_{G}, \psi R_{S}\right)$, where $R_{X}=\left\{\rho_{x} \mid x \in X\right\}$, $\rho_{x}, \psi: V(\Gamma) \rightarrow V(\Gamma)$ are functions defined by $(g, i)^{\rho_{x}}=(g x, i), i=1,2,(g, 1)^{\psi}=\left(g^{-1}, 2\right)$ and $(g, 2)^{\psi}=\left(g^{-1}, 1\right)$.

In the rest of the paper, we use the following remark frequently and without explicit reference.

Remark 1.5. Let $\operatorname{BCay}(G, S)$ be a bi-Cayley graph. Then $\operatorname{BCay}(G, S) \cong \operatorname{BCay}(G, S g)$, for all $g \in G$, see [12, Lemma 2.2]. Also if $1 \in S$ then $\operatorname{BCay}(G, S)$ is connected if and only if $G=\langle S\rangle$, see [11, Lemma 2.7]. Thus, to investigate whether $\operatorname{BCay}(G, S)$ is connected and integral, we may assume that $1 \in S$ and so $G=\langle S\rangle$ if necessary. Also note that, by [112, Lemma 2.1(3)], for all $\alpha \in \operatorname{Aut}(G), \operatorname{BCay}(G, S) \cong \operatorname{BCay}\left(G, S^{\alpha}\right)$. 


\section{Proof of Theorem A}

Let us start with the following easy lemmas.

Lemma 2.1. The groups $\mathbb{Z}_{12}, D_{10}$, and $D_{12}$, have no a connected cubic integral bi-Cayley graph.

Proof. First suppose that $G=\langle a\rangle \cong \mathbb{Z}_{12}$. Suppose, for a contradiction, that there exists $S \subseteq G$ with $|S|=3$ such that $\operatorname{BCay}(G, S)$ is a connected integral graph. By Lemma ㄸ. 4 , $\operatorname{BCay}(G, S) \cong \operatorname{Cay}\left(\langle\psi\rangle R_{G}, \psi R_{S}\right)$ and $\langle\psi\rangle R_{G} \cong D_{24}$. So $D_{24}$ admits a connected cubic integral Cayley graph, which contradicts [ [1, Theorem 1.1].

Now suppose that $G \cong D_{10}=\left\langle a, b \mid a^{5}=b^{2}=(a b)^{2}=1\right\rangle$ and that there exists a subset $S=\left\{s_{1}, s_{2}, s_{3}\right\}$ of $G$ such that BCay $(G, S)$ is connected integral graph. It is well know that $D_{10}$ has a non-trivial linear character $\chi$ such that $\chi\left(a^{i} b^{j}\right)=(-1)^{j}, 0 \leq j \leq 1$ and $0 \leq i \leq 4$. Since BCay $(G, S)$ is connected, 3 is an eigenvalue of multiplicity 1 . Also, by Theorem $\mathbb{\square} \mathbb{\mathbb { l }}, \pm 3$ are eigenvalues of $\operatorname{BCay}(G, S)$ corresponding to the trivial character of $G$. So there exist two elements of $S$, say $s_{1}$ and $s_{2}$ such that $\chi\left(s_{1}\right)=1$ and $\chi\left(s_{2}\right)=-1$. Hence $s_{1}=a^{i}$, and $s_{2}=a^{j} b$ for some $0 \leq i, j \leq 4$. Thus the vertices $(1,1),\left(s_{1}, 2\right),\left(a^{j-i} b, 1\right)$ and $\left(s_{2}, 2\right)$ form a 4 -cycle and so BCay $(G, S)$ has girth $\leq 4$. But by Schwenk's Theorem, there exist exactly two connected cubic integral graphs (up to isomorphisms) with 20 vertices and both of them have girth 6 , see [13] or [[7, Theorem 1], a contradiction.

Finally, suppose that $G \cong D_{12}=\left\langle a, b \mid a^{6}=b^{2}=(a b)^{2}=1\right\rangle$. Suppose, for a contradiction, that there exists a subset $S$ of $G$ such that $\operatorname{BCay}(G, S)$ is connected cubic integral graph. Since $D_{12}$ has a non-trivial linear character $\chi$, where $\chi\left(a^{i} b^{j}\right)=(-1)^{j}, 0 \leq j \leq 1$ and $0 \leq i \leq 5$, similar to the previous case, $\operatorname{BCay}(G, S)$ has girth 4 . On the other hand, by Schwenk's Theorem, there exists exactly one connected cubic integral graph with 24 vertices and this graph has girth 6 , a contradiction.

Lemma 2.2. Let $G$ and $H$ be abelian groups, $S^{-1}=S \subseteq G \backslash\{1\}$ and $T=T^{-1} \subseteq H$. If $\operatorname{Cay}(G, S)$ and $\operatorname{BCay}(H, T)$ are connected and integral, then BCay $(G \times H, R)$, where $R=$ $(S \times\{1\}) \cup(\{1\} \times T)$, is connected and integral.

Proof. Since $\operatorname{Cay}(G, S)$ and $\operatorname{BCay}(H, T)$ are connected, $G=\langle S\rangle$ and $H=\left\langle T T^{-1}\right\rangle$. So $G \times H=$ $\left\langle R R^{-1}\right\rangle$ and $\operatorname{BCay}(G \times H, R)$ is connected. Now let $\chi \in \operatorname{Irr}(G \times H)$. Then there exist $\theta \in \operatorname{Irr}(G)$ and $\mu \in \operatorname{Irr}(H)$ such that $\chi=\theta \times \mu$. Since $\operatorname{Cay}(G, S)$ is integral, $\theta(S) \in \mathbb{Z}$. Also since $T=T^{-1}, \mu(T)=\mu\left(T^{-1}\right)=\overline{\mu(T)}$ and so $\mu(T)=|\mu(T)| \in \mathbb{Z}$ (by Theorem ㅁ. ). Hence $|\chi(R)|=|\theta(S)+\mu(T)|=\theta(S)+\mu(T) \in \mathbb{Z}$. This completes the proof. 
Lemma 2.3. Let $G$ be a finite group. If $\operatorname{Cay}(G, S)$ is connected and integral, then BCay $(G, S \cup$ $\{1\})$ is connected and integral. Thus if $G$ admits a connected integral Cayley graph of valency $k$, then $G$ admits a connected integral bi-Cayley graph of valency $k+1$.

Proof. Put $S_{1}:=S \cup\{1\}$. Then $\left\langle S_{1} S_{1}^{-1}\right\rangle=\langle S\rangle=G$ and so $\operatorname{BCay}\left(G, S_{1}\right)$ is connected. Now let $\rho$ be an irreducible representation of $G$ of degree $d$ and put

$$
\Lambda:=\left[\begin{array}{cc}
0_{d} & \rho\left(S_{1}^{-1}\right) \\
\rho\left(S_{1}\right) & 0_{d}
\end{array}\right] .
$$

Since $\rho(S)=\rho\left(S^{-1}\right)$ we have $\rho\left(S_{1}^{-1}\right)=\rho\left(S_{1}\right)=\rho(S)+I_{d}$, where $I_{d}$ is the identity matrix of order $d$, and so

$$
\Lambda=\left[\begin{array}{cc}
0_{d} & \rho(S)+I_{d} \\
\rho(S)+I_{d} & 0_{d}
\end{array}\right] .
$$

Let $\lambda$ be an eigenvalue of $\rho\left(S_{1}\right)$. Then $\lambda+1$ and $-(\lambda+1)$ are eigenvalues of $\Lambda$.

Since the number of eigenvalues of $\Lambda$ is twice the number of eigenvalues of $\rho(S)$, we conclude that $\mu$ is an eigenvalue of $\Lambda$ if and only if $\mu=\lambda+1$ or $\mu=-(\lambda+1)$ for some eigenvalue $\lambda$ of $\rho(S)$. Since $\operatorname{Cay}(G, S)$ is integral, $\rho(S)$ has integer eigenvalues, and so $\Lambda$ has integer eigenvalues. Hence BCay $\left(G, S_{1}\right)$ is integral, by Theorem $\left.\mathbb{L}\right]$. This completes the proof.

Now we are ready to prove Theorem A. We divide the proof into abelian and non-abelain cases.

\section{Proof of Theorem A (abelian case)}

Let $G$ be a finite abelian group admitting a connected cubic integral bi-Cayley graph $\Gamma=$ $\operatorname{BCay}(G, S)$. By Lemma L4, $\Gamma \cong \operatorname{Cay}\left(\langle\psi\rangle R_{G}, \psi R_{S}\right)$, where $R_{S}=\left\{\rho_{s} \mid s \in S\right\}$ and $\psi$ : $V(\Gamma) \rightarrow V(\Gamma)$ is a function defined by $(x, 1)^{\psi}=\left(x^{-1}, 2\right)$ and $(x, 2)^{\psi}=\left(x^{-1}, 1\right)$. Thus $H:=$ $\langle\psi\rangle R_{G} \cong \mathbb{Z}_{2} \ltimes G$ admits a connected cubic integral Cayley graph. Also $H$ is abelian if and only if $\psi \rho_{g}=\rho_{g} \psi$ for all $g \in G$. Equivalently, $H$ is abelian if and only if $x^{-1} g=g^{-1} x^{-1}$ for all $x, g \in G$. Since $G$ is abelian, we conclude that $H$ is abelian if and only if $G$ is elementary abelian 2-group. Now we have the following cases.

Case I. $G$ is an elementary abelian 2-group. Then $H$ is elementary abelian 2-group, and

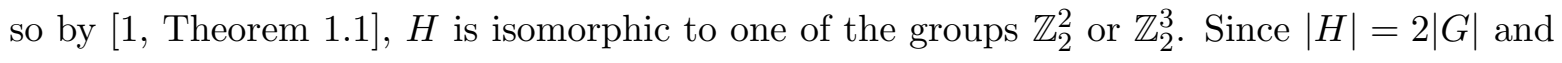
$|G| \geq 3$, we have $G \cong \mathbb{Z}_{2}^{2}$.

Case II. $G$ is not elementary abelian 2-group. Then $H$ is non-abelian and by [I, Theorem 1.1], $H$ is isomorphic to one of the groups $D_{6}, D_{8}, D_{12}, A_{4}, S_{4}, A_{4} \times \mathbb{Z}_{2}, D_{8} \times \mathbb{Z}_{3}, D_{6} \times \mathbb{Z}_{4}$. If $H \cong D_{6}, D_{8}$ or $D_{12}$, then since $G \cong R_{G}$ is a normal subgroup of $H$ of index 2 , we have 
$G \cong \mathbb{Z}_{3}, \mathbb{Z}_{4}$ or $\mathbb{Z}_{6}$, respectively. The case $H \cong A_{4}$ cannot happen, as $A_{4}$ has no subgroup of order 6 . In other cases, we have $|G|=12$ and Lemma $[2$.$] implies that G \cong \mathbb{Z}_{2} \times \mathbb{Z}_{6}$.

To complete the proof, applying Lemma [2.3 and [I, Lemma 2.7], it is enough to prove that $\mathbb{Z}_{2} \times \mathbb{Z}_{6}$ admits a connected cubic integral bi-Cayley graph.

Let $G=\langle a\rangle \times\langle b\rangle \cong \mathbb{Z}_{2} \times \mathbb{Z}_{6}$. Set $S:=\left\{1, b, a b^{2}\right\}$. Then $S S^{-1}=\left\{1, b^{5}, a b^{3}, b, a b^{4}, a b^{2}, a b\right\}$ and $\left\langle S S^{-1}\right\rangle=G$, and so $\operatorname{BCay}(G, S)$ is connected. Now let $\chi \in \operatorname{Irr}(G)$. Then, by Theorem ए.2, there exist $\chi_{1} \in \operatorname{Irr}(\langle a\rangle)$ and $\chi_{2} \in \operatorname{Irr}(\langle b\rangle)$ such that $\chi(S)=1+\chi_{2}(b)+\chi_{1}(a) \chi_{2}(b)^{2}$. Also $\chi_{1}(a)=1$ or -1 and $\lambda:=\chi_{2}(b)=\exp (2 k \pi i / 6)$ for some $k \in\{0,1, \ldots, 5\}$. On the other hand, by inspecting all possible values of $k$, we can see that $\lambda \in\left\{ \pm 1, \pm \frac{1+i \sqrt{3}}{2}, \pm \frac{-1+i \sqrt{3}}{2}\right\}$. If $\chi_{1}(a)=1$, then $|\chi(S)|=\left|1+\lambda+\lambda^{2}\right| \in\{0,1,2,3\}$. If $\chi_{1}(a)=-1$, then $|\chi(S)|=\left|1+\lambda-\lambda^{2}\right| \in\{1,2\}$. Hence, by Theorem $\square$., BCay $(G, S)$ is integral.

\section{Proof of Theorem A (non-abelian case)}

First suppose that $G$ is non-abelian and admits a connected cubic integral bi-Cayley graph $\operatorname{BCay}(G, S)$. By Schwenk's Theorem, all possible connected integral cubic graphs have orders $4,6,8,10,12,20,24,30$, see [1:3] or [7, Theorem 1]. Since BCay $(G, S)$ is of order $2|G|$ and $G$ is non-abelian, $|G|=6,10$, or 12. In fact, by Lemma [2.], $G$ is isomorphic to $S_{3}$, Dic 12 or $A_{4}$.

To complete the proof, applying Lemma [2.3 and [ [1, Lemma 2.7], it is enough to show that both $A_{4}$ and Dic 12 admit a connected cubic integral bi-Cayley graph.

(1) Let $G=A_{4}$. Put $a=(12)(34)$ and $b=(123)$. Then $A_{4}=\langle a, b\rangle$. Now all irreducible representations of $A_{4}$ are

$$
\begin{aligned}
& \rho_{1}: a \mapsto 1, b \mapsto 1, \mapsto \rho_{2}: a \mapsto 1, b \mapsto \omega, \\
& \rho_{3}: a \mapsto 1, b \mapsto \omega^{-1}, \\
& \rho_{4}: a \mapsto\left[\begin{array}{lll}
-1 & 0 & 0 \\
-1 & 0 & 1 \\
-1 & 1 & 0
\end{array}\right], b \mapsto\left[\begin{array}{lll}
-1 & 1 & 0 \\
-1 & 0 & 0 \\
-1 & 0 & 1
\end{array}\right],
\end{aligned}
$$

where $\omega=(-1+i \sqrt{3}) / 2$, see [6, p.111, Example 3]. Set $S:=\left\{1, b, a b^{-1}\right\}$. Then $\rho_{1}(S)=$ $\rho_{1}\left(S^{-1}\right)=3, \rho_{2}(S)=\rho_{2}\left(S^{-1}\right)=\rho_{3}(S)=\rho_{3}\left(S^{-1}\right)=0$,

$$
\rho_{4}(S)=\left[\begin{array}{ccc}
0 & 2 & 0 \\
-1 & 1 & 1 \\
0 & 0 & 2
\end{array}\right], \rho_{4}\left(S^{-1}\right)=\left[\begin{array}{ccc}
1 & -1 & 1 \\
2 & 0 & 0 \\
0 & 0 & 2
\end{array}\right]
$$

So by Theorem $\llbracket$., , the eigenvalues of $\operatorname{BCay}\left(A_{4}, S\right)$ are $0^{[4]},( \pm 1)^{[3]},( \pm 2)^{[6]}$ and $( \pm 3)^{[1]}$, and so $A_{4}$ admits a connected cubic integral bi-Cayley graph. 
(2) Let $G=\operatorname{Dic}_{12}=\left\langle a, b \mid a^{3}=b^{4}=1, b a b^{-1}=a^{2}\right\rangle$. Set $S:=\left\{a, b, b^{2}\right\}$. Then $\left\langle S S^{-1}\right\rangle=G$, which means that $\operatorname{BCay}(G, S)$ is connected. It is easy to see that the following maps are a full list of irreducible representations of $G$

$$
\begin{aligned}
& \rho_{1}: a, b \mapsto 1, \\
& \rho_{2}: a \mapsto 1, b \mapsto-1, \\
& \rho_{3}: a \mapsto 1, b \mapsto-i, \\
& \rho_{4}: a \mapsto 1, b \mapsto i, \\
& \rho_{5}: a \mapsto\left[\begin{array}{ll}
\omega^{2} & 0 \\
0 & \omega
\end{array}\right], b \mapsto\left[\begin{array}{cc}
0 & 1 \\
-1 & 0
\end{array}\right], \\
& \rho_{6}: a \mapsto\left[\begin{array}{ll}
\omega^{2} & 0 \\
0 & \omega
\end{array}\right], b \mapsto\left[\begin{array}{ll}
0 & 1 \\
1 & 0
\end{array}\right],
\end{aligned}
$$

where $\omega=\exp (2 \pi i / 3)$. Now $\rho_{1}(S)=\rho_{1}\left(S^{-1}\right)=3, \rho_{2}(S)=\rho_{2}\left(S^{-1}\right)=1, \rho_{3}(S)=-i=$ $\rho_{4}\left(S^{-1}\right), \rho_{3}\left(S^{-1}\right)=i=\rho_{4}(S)$,

$$
\begin{array}{ll}
\rho_{5}(S)=\left[\begin{array}{cc}
-1+\omega^{2} & 1 \\
-1 & -1+\omega
\end{array}\right], & \rho_{5}\left(S^{-1}\right)=\left[\begin{array}{cc}
-1+\omega & -1 \\
1 & -1+\omega^{2}
\end{array}\right], \\
\rho_{6}(S)=\left[\begin{array}{cc}
1+\omega^{2} & 1 \\
1 & 1+\omega
\end{array}\right], & \rho_{6}\left(S^{-1}\right)=\left[\begin{array}{cc}
1+\omega & 1 \\
1 & 1+\omega^{2}
\end{array}\right] .
\end{array}
$$

It follows from Theorem $\square$. that $0^{[4]},( \pm 1)^{[3]},( \pm 2)^{[6]},( \pm 3)^{[1]}$ are eigenvalues of BCay $\left(\operatorname{Dic}_{12}, S\right)$. Hence Dic 12 admits a connected cubic integral bi-Cayley graph. This completes the proof.

\section{REFERENCES}

[1] A. Abdollahi and E. Vatandoost, Which Cayley graphs are integral? Electron. J. Combin. 16(1) (2009), R122, pp. 1-17.

[2] M. Arezoomand and B. Taeri, On the characteristic polynomial of n-Cayley digraphs, Electron. J. Combin. 20(3) (2013), P57, pp. 1-14.

[3] M. Arezoomand and B. Taeri, Isomorphisms of finite semi-Cayley graphs, Acta Math. Sinica. Eng. Ser., 31(4) (2015), pp. 715-730.

[4] M. Arezoomand and B. Taeri, A classification of finite groups with integral bi-Cayley graphs, Trans. Comb., 4(4) (2015), pp. 55-61.

[5] N. Biggs, Algebraic Graph Theory, Cambridge University Press, (1974).

[6] M. Burrow, Representation theory of finite groups, Academic Press Inc., (1965).

[7] F. C. Bussemaker, D. Cvetković, There are exactly 13 connected, cubic, integral graphs, Univ. Beograd, Publ. Elektrotehn. Fak. Ser. Mat. Fiz., Nos. 544-576 (1976), pp. 43-48 
Alg. Struc. Appl. Vol. 5 No. 2 (2018) 35-43.

[8] P. Diaconis and M. Shahshahani, Generating a random permutation with random transpositions, Z. Wahsch. Verw. Gebiete, 57 (1981), pp. 159-179.

[9] F. Harary and A. J. Schwenk, Which graphs have integral spectra? in "Graphs and Combinatorics (Proc. Capital Conf., George Washington Univ., Washington, D. C., 1973)", Lecture Notes in Mathematics 406. Springer, Berlin, 1974, pp. 45-51.

[10] I. M. Isaacs, Character theory of finite groups, Academic Press, (1976).

[11] W. Jin and W. Liu, A classification of non-abelian simple 3-BCI-groups, Europ. J. Combin., 31 (2010), pp. 1257-1264.

[12] Z. P. Lu, C. Q. Wang, and M.Y. Xu, Semisymmetric cubic graphs constructed from bi-Cayley graphs of $A_{n}$, Ars Combin. 80 (2006), pp. 177-187.

[13] A. J. Schwenk, Exactly thirteen connected cubic graphs have integral spectra, Theory and applications of graphs (Proc. Internat. Conf., Western Mich. Univ., Kalamazoo, Mich., 1976), pp. 516-533, Lecture Notes in Math., 642, Springer, Berlin, (1978).

\section{Majid Arezoomand}

Department of engineering

University of Larestan

Larestan, Iran.

arezoomand@lar.ac.ir

Bijan Taeri

Department of mathematical sciences

Isfahan University of Technology

Isfahan, Iran.

b.taeri@cc.ac.ir 